\title{
Ultrasharp High-aspect-ratio Tetrahedral Molded Tips
}

\author{
Rolf Vermeer, ${ }^{1 *}$ Erwin Berenschot, ${ }^{1}$ Edin Sarajlic, ${ }^{2}$ and Niels Tas ${ }^{1}$ \\ ${ }^{1}$ MESA+ Institute, University of Twente, Drienerlolaan 5, 7522 NB Enschede, The Netherlands \\ ${ }^{2}$ SmartTip B.V., High Tech Factory, Veldmaat 17, 7522 NB Enschede, The Netherlands
}

(Received January 8, 2019; accepted February 19, 2019)

Keywords: microfabrication, anisotropic etching, oxidation sharpening, high-aspect-ratio tip

The fabrication of sharp tips manufactured using silicon or using a silicon mold is a widely used method with a wide range of applications. In this study, we extend our work on the fabrication of tetrahedral molds in (111) silicon and the fabrication of tips based on those molds. We present multiple strategies to make a range of different structures out of silicon-rich nitride, focusing on an approach that takes advantage of oxidation sharpening to improve both the aspect ratio and the tip radius, as well as to make the fabrication process both more efficient and versatile. In this way, single tips and so-called tripod tips with a radius of less than $3 \mathrm{~nm}$ are successfully fabricated and used in an AFM probe to show the functionality of the tips.

\section{Introduction}

The fabrication of sharp tips, made using silicon or made using silicon molds, is investigated extensively for a broad range of applications, such as field emission, atomic force microscopy (AFM), and cell research. ${ }^{(1-8)}$ For typical applications, a robust tip with a high aspect ratio and a small radius is essential. Molded tips, for example made from silicon nitride, diamond-like carbon ${ }^{(9)}$ or metals, ${ }^{(4)}$ are widely used for a range of applications.

Typically, such molded tips are made with a pyramidal pit as the basic mold. ${ }^{(1)}$ However, it has been widely recognized that such molded tips, owing to small imperfections inevitable at the nanometer scale, lead to a bladelike apex rather than a single tip. ${ }^{(10)}$ Furthermore, silicon molds can be sharpened by thermal oxidation; ${ }^{(6,7,11,12)}$ at low temperatures, owing to the stress in the material, the oxidation rate in the vertices of a mold is reduced relative to that on the flat surfaces. ${ }^{(13,14)}$ The combination of imperfect pyramidal pits with oxidation sharpening can lead to the undesired effect of a double tip, where two small tips are close to each other at the apex. ${ }^{(10,15)}$

An alternative approach known in the literature is to stack multiple layers in a smart and well defined way to make a sharpened mold within these layers. This approach, however, suffers from the required lithographic steps and accuracy thereof. The surface roughness plays a prominent role in the definition of the mold as well. ${ }^{(16)}$

To circumvent the potential imperfections of a pyramidal mold, or of a mold made of multiple deposited and lithographically defined layers, we previously presented a method in which a tetrahedral mold is made in (111) silicon. ${ }^{(17)}$ In this way, the tetrahedral silicon crystal *Corresponding author: e-mail: r.vermeer@alumnus.utwente.nl https://doi.org/10.18494/SAM.2019.2255 
structure is used to obtain a fully consistent, symmetric, and predictable mold with three side planes that generally intersect in a single point. With that approach, it has been demonstrated that tetrahedral molded tips are successfully fabricated. In this work, we expand on this approach and investigate further the potential of using such a tetrahedral mold, with a focus on different strategies for and effects of the oxidation sharpening of the molds and thereby the pits made in those molds.

In Sect. 2, the method of mold fabrication described by Vermeer et al. ${ }^{(1)}$ is reiterated. Subsequently, the methods of fabricating tips out of this are described, followed by the results of the fabrication of such tips in Sect. 3. Finally, in Sect. 4, a practical application of an oxidationsharpened tetrahedral tip as an AFM probe, with AFM results, is presented.

\section{Methods}

The fabrication of tetrahedral molded tips consists of two basic processes, namely, the fabrication of a mold and the fabrication of a tip within a mold, which are sequentially discussed here.

\subsection{Mold fabrication}

The fabrication of the mold is schematically shown in Fig. 1 and starts with (111) silicon wafers. The orientation and geometry of $\{111\}$ planes with respect to the wafer surface are critical in the fabrication of the tips. Anisotropic etching techniques ${ }^{(18)}$ play an essential role in the processing.

First, thermal oxidation is performed on a silicon wafer to form a silicon oxide mask, and the silicon oxide layer is patterned using standard lithography with a circular mask opening (a). Second, deep reactive ion etching (DRIE) is used to etch a pit with a conical shape (b). The DRIE process consists of a $\mathrm{SF}_{6}$ plasma for etching and $\mathrm{C}_{4} \mathrm{~F}_{8}$ plasma for deposition. ${ }^{(19)}$ In this etching process, the cycle time and gas flow rate are tuned to obtain a positively tapered pit with a minimized bottom surface area. The last step of the pit fabrication is the development

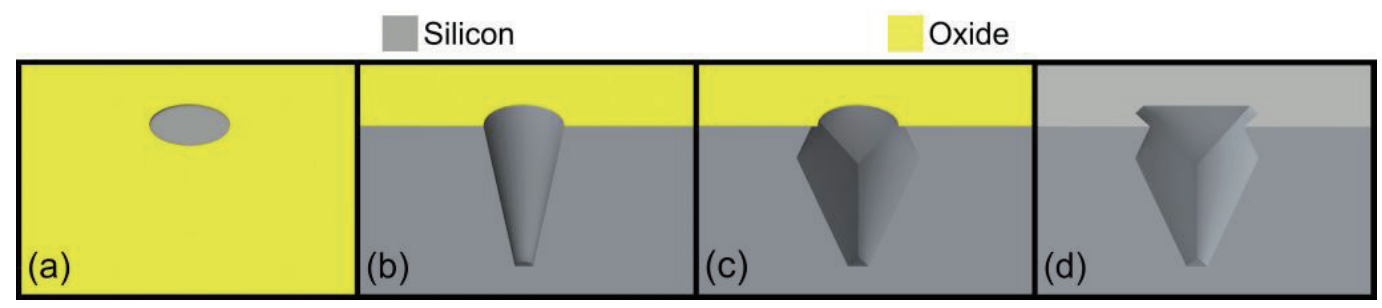

Fig. 1. (Color online) Schematic cross-sectional view of the fabrication process for the tetrahedral molds. (a) A (111) silicon wafer is oxidized and patterned to make (b) a conical pit using a DRIE process followed by (c) anisotropic etching to obtain the tetrahedral shape formed by the (111) crystallographic planes. (d) Finally, the silicon oxide mask is removed to finish the mold. 
of $\{111\}$ planes in the pit using anisotropic etching, tuned for slow, thus controlled, etching and a low etching ratio of $\{111\}$ planes with respect to the other crystal planes (c). Inevitably, the DRIE process will always result in pits with a small bottom surface area. After the anisotropic etching, this will unpreventably result in a triangular bottom surface in the pits. After the anisotropic etching, the silicon oxide mask is removed to complete the mold fabrication (d).

\subsection{Tip machining}

With such molds, several methods of fabricating tips can be employed. We previously presented a general process to develop tips using the molds. ${ }^{(17)}$ Starting with a silicon wafer with tetrahedral pits (a), the process comprises filling the mold with a fill-up layer, conformally deposited by the low-pressure chemical vapour deposition (LPCVD) of tetraethyl orthosilicate (TEOS). Owing to the geometry of $\{111\}$ side planes in combination with the bottom (111) surface, the size of the bottom surface decreases with increasing layer thickness. Subsequently, the device layer that forms the tips is deposited, for example, using LPCVD silicon-rich nitride (SiRN). To properly release the tips from their mold and remove the fill-up layer, it is essential that materials are chosen such that a high selectivity exists between the material of the tips and those of the mold and fill-up layer. As silicon can be removed with potassium hydroxide $(\mathrm{KOH})$ or tetramethyl ammonium hydroxide (TMAH) etching steps and TEOS with buffered hydrofluoric acid (BHF) with high selectivity with respect to SiRN, this combination of layers has been chosen.

This general process results in sharp tips with a tetrahedral shape, following the $\{111\}$ side planes of the mold. To further investigate the possibilities that this method provides, several modifications are made to this general process to alter the resulting tips.

\subsubsection{Direct mold - flat tip apex}

Firstly, using a truncated tetrahedral pit directly as a mold, thus without a fill-up layer, will result in a tip with a triangular flat apex, as shown in Fig. 1(d). By tuning the tapering and sharpness of the pit, either by tuning the DRIE or KOH etching steps, the size of the triangular flat apex is tuned.

\subsubsection{Conformal filling and oxidation - oxidation sharpened tip}

Secondly, given the mold filled with a TEOS fill-up layer, by thermal oxidation at a relatively low temperature, both the TEOS layer and the underlying silicon are annealed and, most importantly, oxidized. This process results in the oxidation sharpening of the mold, as schematically shown in Fig. 2. The rest of the process remains the same and results in an oxidation-sharpened SiRN three-plane tip, which has an increased aspect ratio towards the apex compared with the nonsharpened tip. 


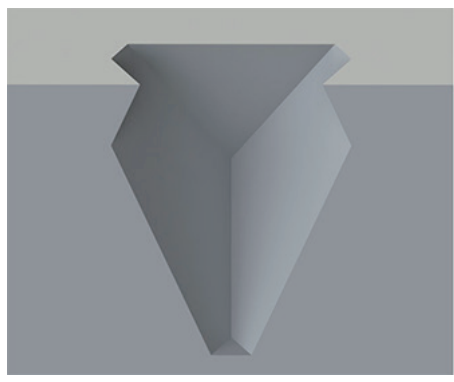

(a)

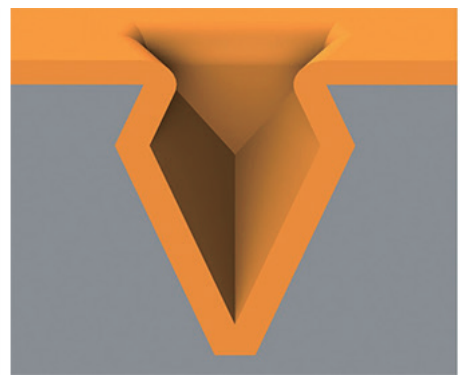

(b)

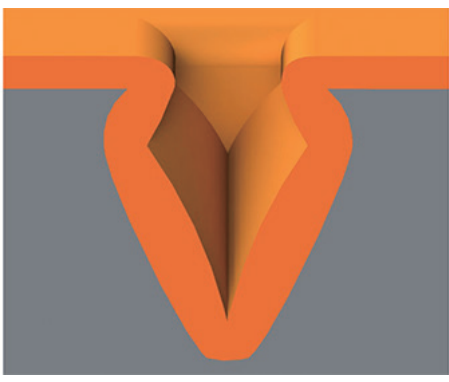

(c)

Fig. 2. (Color online) Schematic cross-sectional drawing of the thermal oxidation of the TEOS fill-up layer, showing the silicon mold (a), the nonsharpened mold with the TEOS fill-up layer (b), and the result of the thermal oxidation of the TEOS and the underlying silicon, resulting in the sharpening of the mold (c).

\subsubsection{Mold oxidation - tripod tip and single tip}

Thirdly, the truncated tetrahedral pit is not filled up with an LPCVD layer of TEOS, but instead is directly thermally oxidized at a relatively low temperature, as shown in Fig. 1(d). In that case, the shape of the mold will depend strongly on the thickness of the silicon oxide layer with respect to the size of the bottom surface of the pit, as schematically depicted in Fig. 3. Owing to the triangular bottom surface and the oxidation thinning in the convex corners, the oxidation process will initially result in a mold with three small pits in the corners of the triangular bottom surface. By further oxidization, the sidewalls of the pit will form a neck in the pit, which will ultimately result in a sharp pit.

This will subsequently yield a SiRN tip with three tips, the so-called tripod tip, after longer oxidation, or a single relatively shorter single SiRN tip will be formed, as schematically shown in Fig. 4.

\section{Results and Discussion}

The DRIE process used to etch the tapered pits in the (111) silicon is a fast-switching deposition-limited Bosch process performed in an Adixen AMS100 SE at $-40{ }^{\circ} \mathrm{C}$. The tapering is achieved by passivation using $175 \mathrm{sccm} \mathrm{C}_{4} \mathrm{~F}_{8}$ for $1.0 \mathrm{~s}$, which is balanced with etching using $150 \mathrm{sccm} \mathrm{SF}_{6}$ for $0.5 \mathrm{~s}$, both at an ICP of $1500 \mathrm{~W}$ and a CPP of $60 \mathrm{~W}$, with a duty cycle of $10 \%$. In this way, the depth of the pit and the size of the bottom surface will be optimized after anisotropic etching. To obtain the smallest possible bottom surface, the $\mathrm{SF}_{6}$ etching is reduced as the etching time progresses, which in turn results in a lower etch rate and thus a pit that is relatively shallow. Eventually, this will in the end result in a relatively small tip. On the other hand, if a relatively large tip is required, thus a relatively deep tapered pit, the $\mathrm{SF}_{6}$ etching remains constant over the entire etching process, which as a consequence also results in a larger bottom surface. 


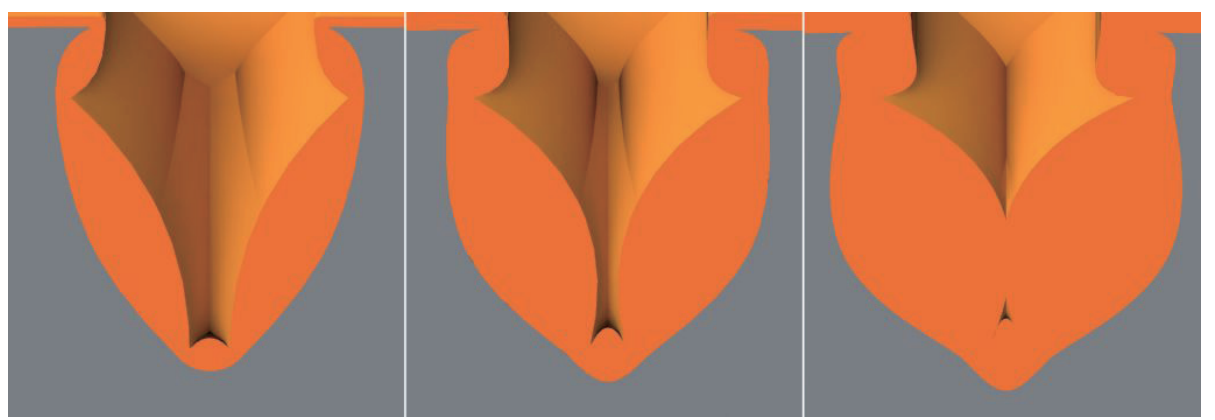

(a)

(b)

(c)

Fig. 3. (Color online) Schematic cross-sectional drawing of the thermal oxidation of the silicon tetrahedral mold, which initially results in a mold with three small pits (a). Longer oxidation results in an increasingly thinner neck (b), which ultimately sharpens to a single pit (c).

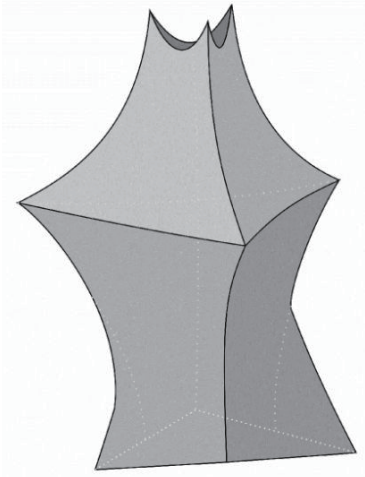

(a)

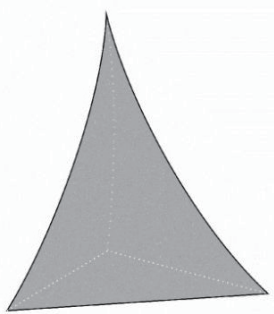

(b)

Fig. 4. Schematic drawing of the filling of the molds obtained by thermal oxidation of silicon, which will result in a tripod tip for short oxidation duration (a) and in a single tip for longer oxidation duration (b).

For anisotropic etching, a specially prepared solution of $\mathrm{KOH}$ is used. As stated earlier, the $\mathrm{KOH}$ etching is aimed at controlled etching and a low etching ratio of the $\{111\}$ planes with respect to the other crystal planes, such that the $\{111\}$ side planes are well developed with a minimal bottom (111) surface. Commonly used $\mathrm{KOH}$ etching solutions typically are aimed at the fast etching of relatively large structures and thus have etch rates for the $\langle 001\rangle$ and $\langle 011\rangle$ directions on the order of several hundreds of nanometers to over one micrometer per minute, depending on temperature and etchant concentration, ${ }^{(20)}$ and the etch rate in the $\langle 111\rangle$ directions is several nanometers to tens of nanometers per minute. ${ }^{(21,22)}$ However, for this application, much lower etch rates are required. For well-tapered pits after the DRIE process, no more than $500 \mathrm{~nm}$ of silicon has to be etched in the $\langle 001\rangle$ and $\langle 011\rangle$ directions. To controllably achieve this, a dedicated $\mathrm{KOH}$ solution, consisting of $60 \mathrm{wt} \% \mathrm{KOH}$ in water at $20^{\circ} \mathrm{C}$, is prepared. This solution is characterized for (001) silicon, (111) silicon, and thermally grown silicon oxide, as this oxide is used as the masking material. The etch rate is measured over several days of 
etching, resulting in average etch rates of $0.46 \mu \mathrm{m} / \mathrm{h}$ in the $\langle 001\rangle$ direction, $3.2 \mathrm{~nm} / \mathrm{h}$ in the $\langle 111\rangle$ direction, and $0.55 \mathrm{~nm} / \mathrm{h}$ for thermally grown silicon oxide. The etch rate ratio of the $\langle 001\rangle$ and $\langle 111\rangle$ directions is therefore $R_{001}: R_{111}=142: 1$, and this, together with the sufficiently low etch rates for both silicon and silicon oxide, results in an etching solution that gives excellent control over the $\mathrm{KOH}$ etching process, without degrading the silicon oxide mask.

Using the combination of the dedicated DRIE and $\mathrm{KOH}$ etching processes, starting from a 5 - $\mu \mathrm{m}$-diameter circular mask opening, pits have been obtained with the length of the sides of the triangular bottom surfaces as small as $50 \mathrm{~nm}$, as depicted in Fig. 5. The pits are, depending on the balancing of the DRIE process, as described earlier, estimated to be between 8 and 15 $\mu \mathrm{m}$ deep.

On the basis of these molds, all the mentioned different types of tips have been fabricated. By not filling the silicon molds with a fill-up layer, a tip is made with a flat triangular top surface. The size of the triangular top surface is tuned by balancing the DRIE process and changing the $\mathrm{KOH}$ etching time; in this way, flat SiRN tips are made with flat surfaces with side lengths ranging from $60 \mathrm{~nm}$ to $1 \mu \mathrm{m}$, as shown in Fig. 6 . As the etching of the pit is very well controlled, especially by the very slow $\mathrm{KOH}$ etching, this process results in very well controlled top surface sizes with a high uniformity over the wafer, where the typical nonuniformity of the tip size is less than $10 \%$. Furthermore, as the mold has a perfect crystalline (111) plane as its bottom surface, the top surface of the tip is extremely smooth. The smoothness of the tip surface could, for example, be of great benefit in friction and contact mechanics probing. ${ }^{(23,24)}$

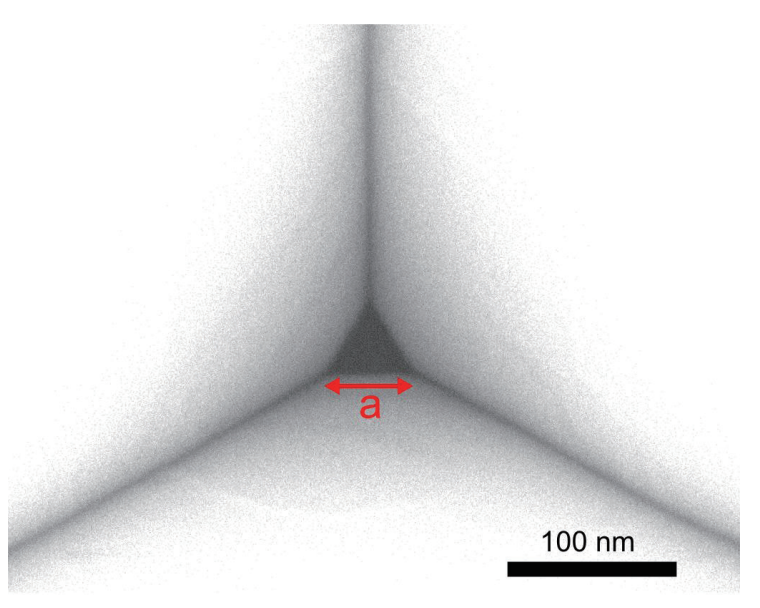

Fig. 5. (Color online) Top-view scanning electron microscopy (SEM) image of a pit in silicon after $\mathrm{KOH}$ etching, showing the three sides of the tetrahedral pit and the bottom surface in the center. Furthermore, the length of the sides of the triangle, the parameter a as used in Eq. (2), is indicated by the arrow.

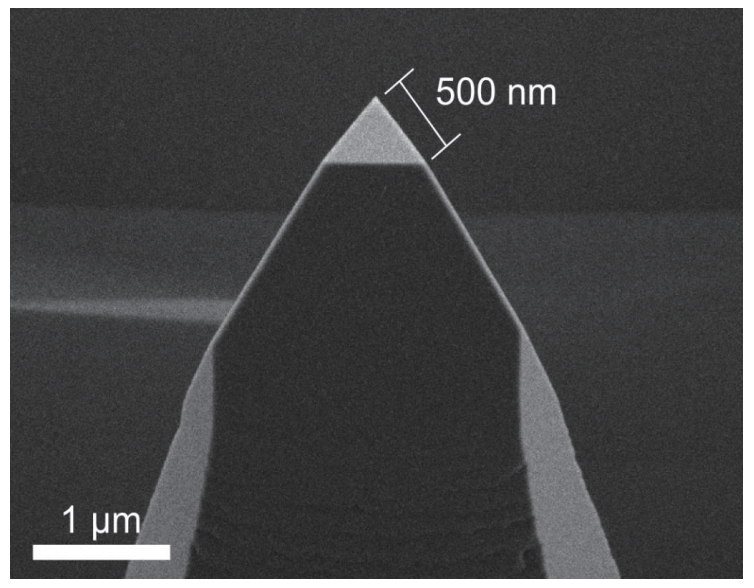

Fig. 6. SEM image of a silicon nitride tip with a flat top surface and a side length of approximately 500 nm. 
We previously presented the results of conformally filling the mold with a layer of TEOS and subsequently depositing the SiRN device layer. ${ }^{(17)}$ In addition to the work presented there, it is important to note that for this process to result in a sharp tip, the conformal deposition of the fill-up TEOS layer plays a crucial role. If for some reason the thickness of the fill-up layer on the inclined surface, $t_{i n c l}$, is a factor $X$ less, which is practically common, ${ }^{(25)}$ the ratio of the thickness on the inclined surface to that on the flat surfaces is given by Eq. (1).

$$
t_{\text {incl }}=X \times t_{\text {flat }}
$$

The minimum thickness of the fill-up layer, $t_{\min }$, over the side length of the initial bottom triangle, $a$, for this specific geometry is easily derived using the properties of the (111) planes in silicon and standard trigonometry. The minimum thickness of the fill-up layer in that situation is described by Eq. (2) and depicted in Fig. 7.

$$
t_{\min }=\frac{\sqrt{6}}{9 X-3} a
$$

Typically, the annealing of TEOS is used to densify the TEOS layers. ${ }^{(26)}$ In this case, however, that would lead to undesired effects, such as the rounding of the TEOS layer at the bottom of the pit, which would result in a rounded tip. Therefore, we instead use the thermal oxidation of the TEOS-filled mold. The thermal oxidation of the underlying silicon results in the further oxidation of the TEOS layer and the underlying silicon. For this process, a relatively low temperature dry oxidation is performed at $1050{ }^{\circ} \mathrm{C}$ to prevent the viscous flow of the oxide

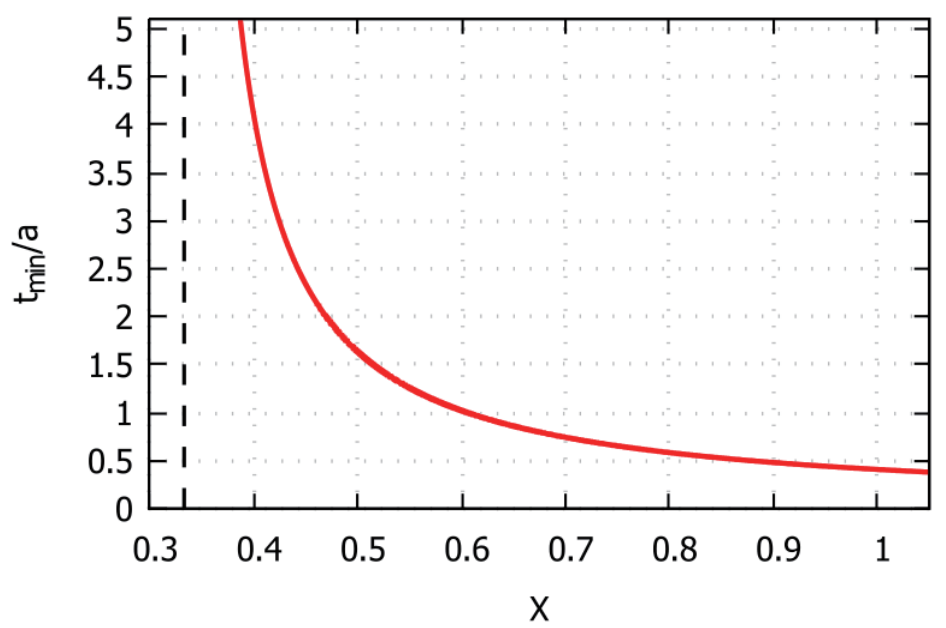

Fig. 7. (Color online) Minimal thickness $t_{\min }$ that is required to completely fill the mold relative to the side length of the original bottom surface, $a$, versus the ratio of the thickness of the "conformally" grown fill-up layer on the inclined surface to that on the flat surface, $X$, as given by Eq. (1). 
in the concave corners. This temperature is, however, sufficiently high to obtain a sufficiently thick oxide layer. Owing to the reduced oxidation rate in concave corners and edges in silicon, ${ }^{(27)}$ and as the combined thickness of the TEOS fill-up layer at the edges and bottom of the pit is larger, which further decreases the oxidation rate at the edges and bottom of the pit, the TEOS-filled mold is oxidation-sharpened. ${ }^{(28)}$ This in turn results in a sharpened SiRN tip with an increased aspect ratio with respect to the unoxidized mold. SEM images of the resulting SiRN tip are shown in Fig. 8.

Apart from the very high aspect ratio of the tip, the radius of the oxidation-sharpened SiRN tetrahedral tip is determined on the basis of and limited by SEM imaging. To this extent, edge detection is used to determine the theoretical extrapolation of the two sides of the tip in the SEM image. Subsequently, a circle is measured touching both sides of the tip, such that the entire circle falls within the tip, as schematically depicted in Fig. 9. On the basis of this, the radius of the oxidation-sharpened SiRN tetrahedral tip is estimated to be less than $3 \mathrm{~nm}$.
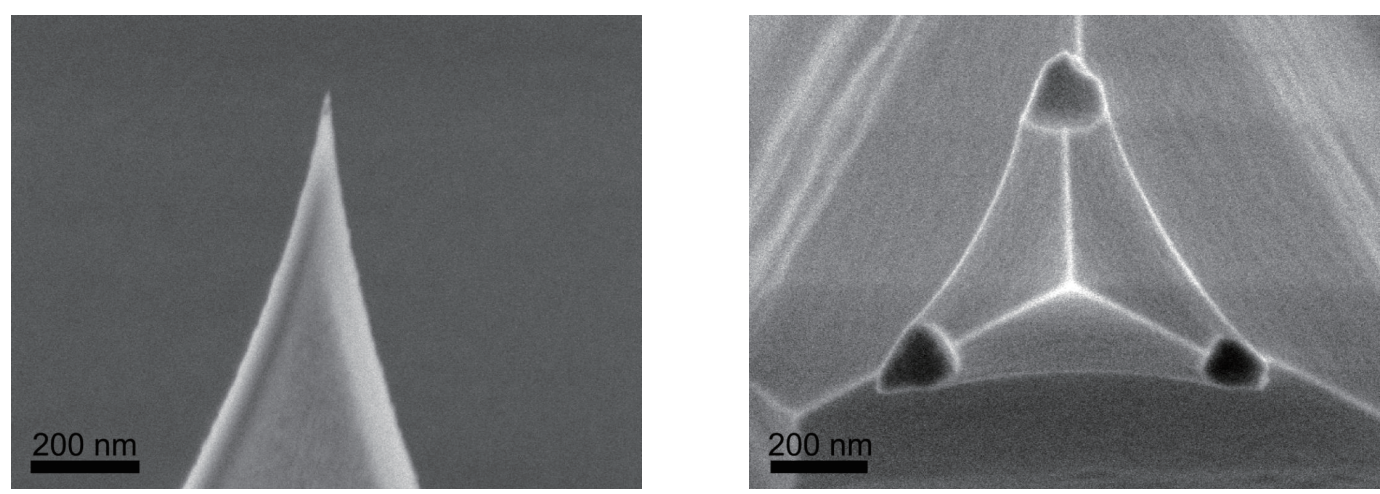

Fig. 8. SEM image of a SiRN tip that was molded in a thermally oxidized TEOS mold, which results in an oxidation-sharpened tip with a radius of less than $3 \mathrm{~nm}$.

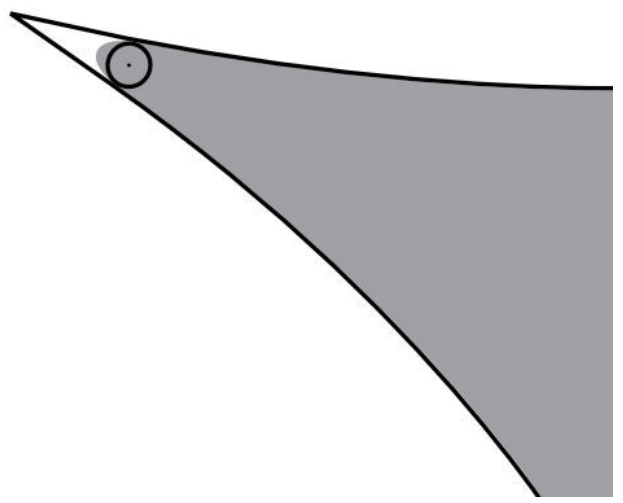

Fig. 9. Schematic representation of the tip radius determination procedure. In grey, the tip as imaged by SEM is depicted, and in black, the edges are shown, after which the radius is determined with the inscribed circle, also shown in black. 
When no conformal fill-up layer is deposited in the tetrahedral mold and the mold is subsequently thermally oxidized, the shape of the resulting tip strongly depends on the oxidation time and temperature with respect to the dimensions of the pit. In both of the two following cases, oxidation was performed for $12 \mathrm{~h}$ at $1050{ }^{\circ} \mathrm{C}$.

In the first case, the DRIE process and $\mathrm{KOH}$ etching time were chosen to obtain relatively big bottom surfaces of approximately $1.2 \mu \mathrm{m}$. As the oxidation of silicon for $12 \mathrm{~h}$ at $1050{ }^{\circ} \mathrm{C}$ results in approximately $400 \mathrm{~nm}$ oxide on flat (111) planes, the oxidation sharpening of the bottom surface occurs in such a way that a mold with three small pits emerges. The resulting tripod tip therefore also consists of 3 small tips in the same plane, as depicted on the left side in Fig. 10 by SEM imaging.

In the second case, starting with a similar size of the bottom surface of the pit, a much higher level of oxidation was performed for $36 \mathrm{~h}$. This still results in a tripod tip with three tips, but with a much higher aspect ratio and a more pronounced shape. Compared with the first case, the tripod is considerably less flat, as shown on the right side in Fig. 10.

In the third case, the DRIE and $\mathrm{KOH}$ etching processes were tuned to minimize the bottom surface to less than $150 \mathrm{~nm}$. Then, thermal oxidation for $12 \mathrm{~h}$ at $1050{ }^{\circ} \mathrm{C}$ results in the congregation of the sides of the mold, in which a neck is formed in the mold that eventually results in a sharp tip. The SiRN tip formed by filling the mold is shown by SEM imaging in Fig. 11. From the SEM imaging and using the same analysis methodology described before, the tip radius for direct thermal oxidation is estimated to be $8 \mathrm{~nm}$, which is not as small as those in the above-mentioned fabrication methods. The benefit of this tip, however, lies in a simplified process flow, which does not rely on methods of conformal deposition with LPCVD, while still resulting in an oxidation-sharpened high-aspect-ratio molded tip with a very sharp apex.

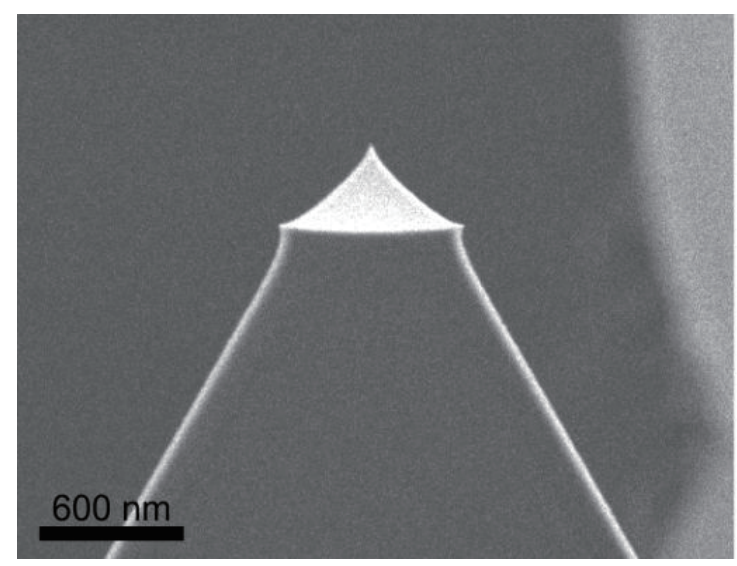

(a)

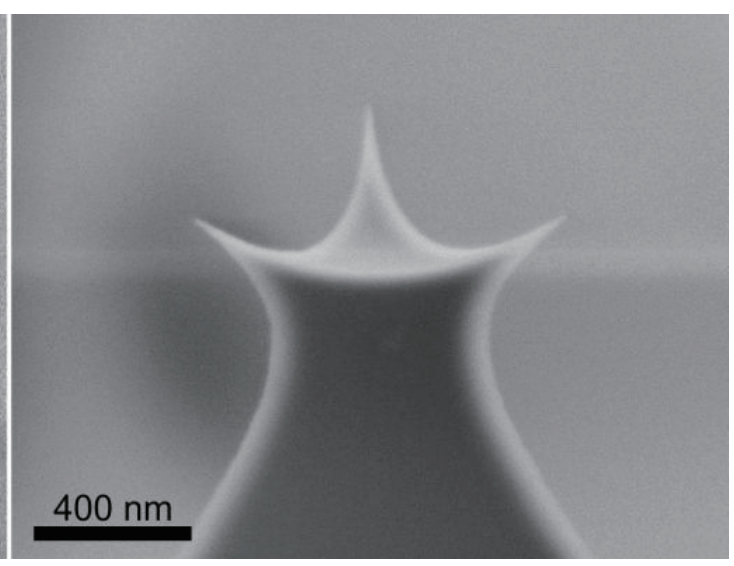

(b)

Fig. 10. SEM image of a silicon nitride tip originating from a silicon mold, which was directly thermally oxidized at low temperature. (a) The result is shown for a relatively short amount of oxidation time (relative to the size of the bottom surface of the pit), resulting in a relatively flat tripod tip with three small sharp tips. (b) The result is shown for a relatively long oxidation time, but still short enough to end up with three tips, with a significantly higher aspect ratio. 


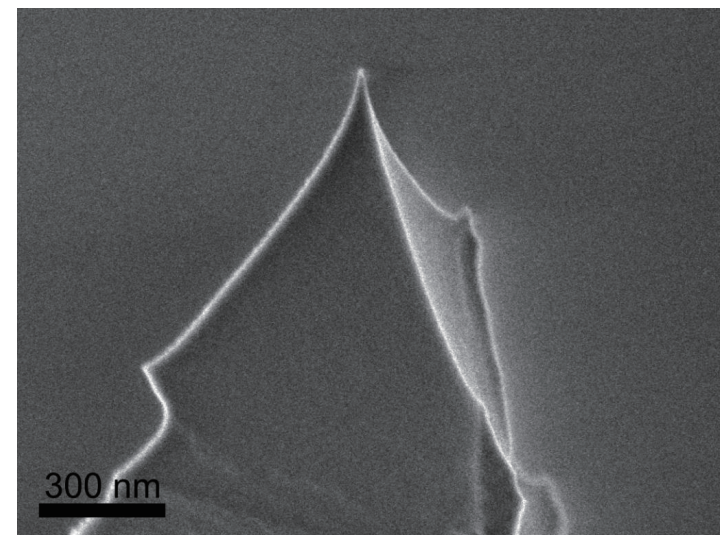

Fig. 11. SEM image of a silicon nitride tip fabricated from a directly thermally oxidized silicon mold, where oxidation was performed for a relatively long time, resulting in a sharpened $\mathrm{SiRN}$ tip with an estimated radius of $8 \mathrm{~nm}$.

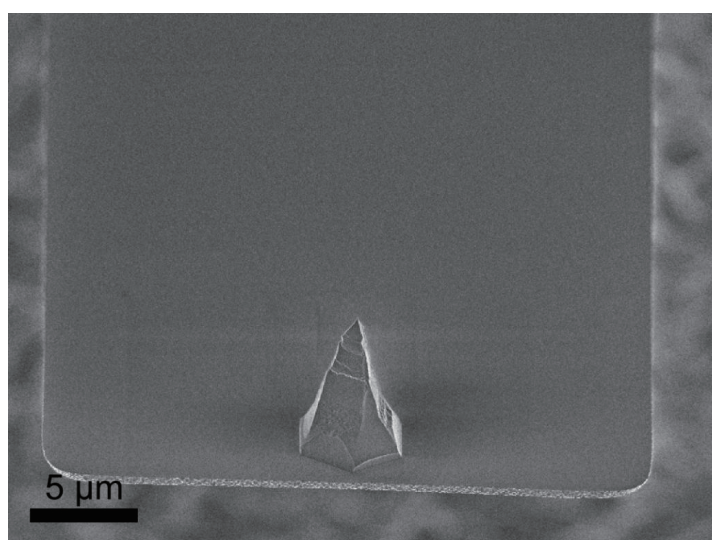

Fig. 12. SEM image of the silicon nitride cantilever with the tip at the end of the cantilever.

\section{AFM Experiments}

For the fabrication of cantilever probes, as previously published, ${ }^{(17)}$ the cantilever width, thickness, and length of $30 \mu \mathrm{m}, 600 \mathrm{~nm}$, and $100 \mu \mathrm{m}$, respectively, have been chosen, such that the cantilever will have the theoretical stiffness and resonance frequency of $0.5 \mathrm{~N} / \mathrm{m}$ and $110 \mathrm{kHz}$, respectively. ${ }^{(29)}$ This is in the range of typical AFM tapping mode probes and therefore the dimensions have been chosen as such.

As explained in Ref. 17, the complete removal of the silicon wafer after bonding to the glass carrier wafer requires extra attention as a (111) silicon wafer is used. The removal process in this case deviates from what is common; as in most situations, the silicon wafer, which has to be removed at some point to expose the tip, is a (001) wafer. A (001) wafer is easily removed by etching for $10-15 \mathrm{~h}$ in TMAH or $\mathrm{KOH}$. The fact that we have used a (111) wafer here means that the wafer surface etches extremely slowly during anisotropic etching and that the complete removal of the silicon wafer would take several weeks. Therefore, after defining the cantilever and chip in the stack of deposited layers, another lithographic step is used to define rectangular openings through which wafer-through DRIE is performed. These holes in and completely through the wafer will expose the vertical planes in the (111) wafer, which are not formed along slow etching directions, and can therefore be etched much faster. In this way, the total etching time will be reduced to $8-12 \mathrm{~h}$, which is common for this procedure.

After the removal of the silicon wafer and oxide layers, where applicable, the tip and cantilever are exposed as shown in the SEM image in Fig. 12. The probes are then separated by breaking the glass into single probes through the breaking grooves that have been diced, whereby the fabrication of the probes is successfully completed. Owing to the geometry of the tip, the fabrication thereof is very robust and the yield therefore is on the order of $90 \%$. 


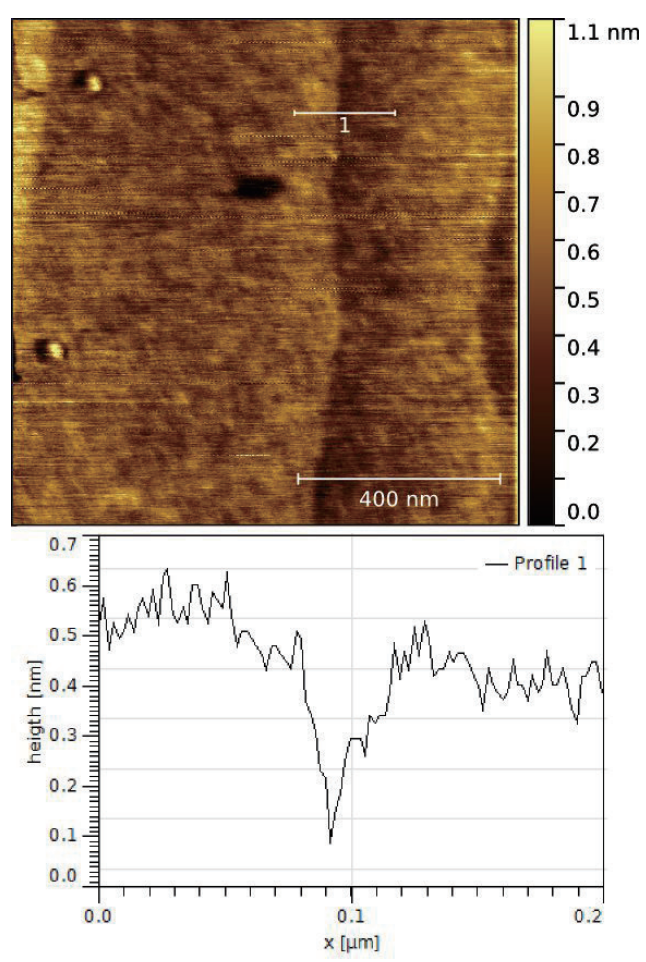

Fig. 13. (Color online) AFM measurements of a (111) silicon sample with atomic steps, with the SiRN tip obtained from a mold with thermally oxidized TEOS inside. The scale bar represents $400 \mathrm{~nm}$. Underneath the topography image, a line profile is shown, as indicated by the white line in the topography image indicated by the number 1.

The sharpest tips based on the SEM analysis discussed before, being the SiRN tips fabricated from a thermally oxidized TEOS mold, are used to perform AFM measurements. The measurements are performed on a (111) silicon sample with atomic steps in the contact mode. Multiple tips are used to perform a number of AFM measurements. A typical topography image and a typical line image of one of those measurements are shown in Fig. 13.

In the topography image of the measurement, multiple atomic steps are clearly observed, and the roughness of the sample is also visible. The line profile confirms this observation and on the basis of the analysis of this measurement and the observed features, the tip radius is determined to be less than $4 \mathrm{~nm}$ in one direction, which is in good agreement with the SEM analysis.

\section{Conclusions}

We successfully demonstrated a method of fabricating tetrahedral molds with perfect $\{111\}$ planes as side walls, which we controllably tune in depth and bottom surface size. To this extent, dedicated DRIE and anisotropic etching have been developed, which allow a high degree of control over the shape of the molds. The tapering of the mold prepared by DRIE is controlled by limiting the etching over time during the Bosch process. The etching of the 
(111) planes is limited by using a highly selective and highly concentrated $\mathrm{KOH}$ solution for anisotropic etching at room temperature, resulting in a 142:1 etch speed ratio when comparing etching in the $\langle 001\rangle$ direction to that in the $\langle 001\rangle$ direction.

By using such molds, several methods have been shown to fabricate tips of various shapes ranging from flat tips to tripod and ultrasharp single tips. The choices made in the layers grown inside a mold play a crucial role here. In this way, flat triangular tips with a side length ranging from $60 \mathrm{~nm}$ to $1 \mu \mathrm{m}$ are fabricated. By the oxidation of the conformally filled mold, the perfectly tetrahedral tips are sharpened to achieve an extra high aspect ratio. On the basis of SEM imaging, it is estimated that a tip radius of less than $3 \mathrm{~nm}$ is achieved.

Furthermore, the direct low-temperature oxidation of the mold results in a tripod tip with three small sharp tips for a relatively short oxidation time and a sharpened single tip with a typical tip radius of $8 \mathrm{~nm}$ for a relatively long oxidation time.

Given the successful development of methods of fabricating several different types of molded tips, a procedure to create a complete AFM probe has been developed, which has successfully been used for AFM imaging that yields topography images with both high lateral and spatial resolutions. The AFM images obtained confirm the SEM-based estimate of the tip radius.

\section{Acknowledgments}

This work was supported by NanoNextNL, a micro- and nanotechnology consortium of the Government of the Netherlands and 130 partners. The authors would like to thank Mark Smithers and Henk van Wolferen $\left(\mathrm{MESA}^{+}\right.$NanoLab, University of Twente) for their assistance with SEM imaging, and Martin Siekman (MESA ${ }^{+}$Institute, University of Twente) for his assistance with AFM imaging.

\section{References}

1 T. R. Albrecht, S. Akamine, T. E. Carver, and C. F. Quate: J. Vac. Sci. Technol., A 8 (1990) 3386. https://doi. org/10.1116/1.576520

2 R. B. Marcus, T. S. Ravi, T. Gmitter, K. Chin, D. Liu, W. J. Orvis, D. R. Ciarlo, C. E. Hunt, and J. Trujillo: Appl. Phys. Lett. 56 (1990) 236. https://doi.org/10.1063/1.102841

3 A. Boisen, O. Hansen, and S. Bouwstra: J. Micromech. Microeng. 6 (1996) 58. https://doi.org/10.1088/0960$1317 / 6 / 1 / 012$

4 A. Boisen, J. P. Rasmussen, O. Hansen, and S. Bouwstra: Microelectron. Eng. 30 (1996) 579. https://doi. org/10.1016/0167-9317(95)00314-2

5 T. Hantschel, R. Stephenson, T. Trenkler, P. De Wolf, and W. Vandervorst: Proc. SPIE 3680 (1999) 994. https:// doi.org/10.1117/12.341168

6 R. J. Grow, S. C. Minne, S. R. Manalis, and C. F. Quate: J. Microelectromech. Syst. 11 (2002) 317. https://doi. org/10.1109/JMEMS.2002.800924

7 K. Sun, J. Y. Lee, B. Li, W. Liu, C. Miao, Y.-H. Xie, X. Wei, and T. P. Russell: J. Appl. Phys. 108 (2010) 036102. https://doi.org/10.1063/1.3437079

8 X. Zhang, X. Yu, T. Li, and Y. Wang: Microsyst. Technol. 24 (2018) 2913. https://doi.org/10.1007/s00542-0173687-z

9 Y.-S. Kim, C. Sunyong Lee, W.-H. Jin, S. Jang, H.-J. Nam, and J.-U. Bu: Sens. Mater. 17 (2005) 57.

10 E. Sarajlic, C. Yamahata, and H. Fujita: Microelectron. Eng. 84 (2007) 1419. https://doi.org/10.1016/ j.mee.2007.01.250

11 A. Folch, M. S. Wrighton, and M. A. Schmidt: J. Microelectromech. Syst. 6 (1997) 303. https://doi. org $/ 10.1109 / 84.650126$ 
12 D. P. Burt, P. S. Dobson, L. Donaldson, and J. M. R. Weaver: Microelectron. Eng. 85 (2008) 625. https://doi. org/10.1016/j.mee.2007.11.010

13 S. Akamine and C. F. Quate: J. Vac. Sci. Technol., B 10 (1992) 2307. https://doi.org/10.1116/1.586059

14 H. He, J. Zhang, J. Yang, and F. Yang: Microsyst. Technol. 23 (2017) 1799. https://doi.org/10.1007/s00542-0162941-0

15 N. Moldovan, Z. Dai, H. Zeng, J. A. Carlisle, T. D. B. Jacobs, V. Vahdat, D. S. Grierson, J. Liu, K. T. Turner, and R. W. Carpick: J. Microelectromech. Syst. 21 (2012) 431. https://doi.org/10.1109/JMEMS.2011.2174430

16 A. Hattori: US Patent 20010001225, 2001.

17 R. Vermeer, J. W. Berenschot, E. Sarajlic, N. R. Tas, and H. V. Jansen: 14th IEEE Int. Conf. Nanotechnology (IEEE, Toronto, Canada, 2014) 229-233. https://doi.org/10.1109/NANO.2014.6967957

18 R. E. Oosterbroek, J. W. Berenschot, H. V. Jansen, A. J. Nijdam, G. Pandraud, A. van den Berg, and M. C. Elwenspoek: J. Microelectromech. Syst. 9 (2000) 390. https://doi.org/10.1109/84.870065

19 H. V. Jansen, M. J. de Boer, S. Unnikrishnan, M. C. Louwerse, and M. C. Elwenspoek: J. Micromech. Microeng. 19 (2009) 033001. https://doi.org/10.1088/0960-1317/19/3/033001

20 M. Shikida, K. Sato, K. Tokoro, and D. Uchikawa: 12th IEEE Int. Conf. Micro Electro Mechanical Systems (1999) 315-320. https://doi.org/10.1109/MEMSYS.1999.746845

21 D. L. Kendall: Appl. Phys. Lett. 26 (1975) 195. https://doi.org/10.1063/1.88113

22 M. Elwenspoek: J. Micromech. Microeng. 6 (1996) 405. https://doi.org/10.1088/0960-1317/6/4/007

23 Y. Ando and J. Ino: Sens. Actuators, A 57 (1996) 83. https://doi.org/10.1016/S0924-4247(97)80098-2

24 R. Buzio, C. Boragno, and U. Valbusa: Wear 254 (2003) 917. https://doi.org/10.1016/S0043-1648(03)00247-3

25 P. McCann, K. Somasundram, S. Byrne, and A. Nevin: Micromachining and Microfabrication, J. M. Karam and J. A. Yasaitis Eds. (SPIE, San Francisco, CA, USA, 2001) 329-340. https://doi.org/10.1117/12.442963

26 C. Orfescu, C. Pavelescu, and M. Badila: J. Mater. Sci. 25 (1990) 1366. https://doi.org/10.1007/BF00585451

27 R. B. Marcus and T. T. Sheng: J. Electrochem. Soc. 129 (1982) 1278. https://doi.org/10.1149/1.2124118

28 T. S. Ravi, R. B. Marcus, and D. Liu: J. Vac. Sci. Technol., B 9 (1991) 2733. https://doi.org/10.1116/1.585680

29 R. C. Hibbeler: Statics and Mechanics of Materials (Pearson, Singapore, 2011) 3rd ed. 